\title{
Evaluation of SNR for AWGN, Rayleigh and Rician Fading Channels Under DPSK Modulation Scheme with Constant BER
}

\author{
Deepak K. Chy ${ }^{1}$, Md. Khaliluzzaman ${ }^{2}$ \\ ${ }^{1}$ Department of Electrical \& Electronic Engineering, University of Information Technology \& Sciences (UITS), Dhaka, Bangladesh \\ ${ }^{2}$ Department of Computer Science \& Engineering, University of Information Technology \& Sciences (UITS), Dhaka, Bangladesh
}

\section{Email address:}

dk_chy53@yahoo.de (D. K. Chy), khalil_021@yahoo.co.in (M. Khaliluzzaman)

\section{To cite this article:}

Deepak K. Chy, Md. Khaliluzzaman. Evaluation of SNR for AWGN, Rayleigh and Rician Fading Channels Under DPSK Modulation Scheme with Constant BER. International Journal of Wireless Communications and Mobile Computing. Vol. 3, No. 1, 2015, pp. 7-12.

doi: 10.11648/j.wcmc.20150301.12

\begin{abstract}
With the growing demand in modern communication, it has become necessary to give better and efficient service to users by using better technique. Technique such as Amplitude Shift Keying (ASK), Frequency Shift Keying (FSK), Phase Shift Keying (PSK), Differential Phase shift Keying (DPSK) and Quadrature Amplitude Modulation (QAM) are very important parts of the implementation of modern communications systems in which DPSK is the simplest and most robust of all techniques. In this paper, evaluation of SNR in terms of constant bit error rate is performed on AWGN, Rayleigh and Rician fading channels. Among these channels, Rician is showing better performance as compared to AWGN and Rayleigh.
\end{abstract}

Keywords: DPSK, SNR, BER, AWGN, Rayleigh, Rician

\section{Introduction}

Mobile communications and wireless network have experienced massive growth and commercial success in the recent years. However, the radio channels in mobile radio systems are usually not amiable as the wired one. Unlike wired channels that are stationary and predictable whereas wireless channels are extremely random and time-variant.

In general Communication can be defined simply as 'sending and receiving messages', or 'the transmission of messages from one person to another'. Effective communication occurs only when the receiver understands the exact message sent by the transmitter [1].

The next generation of wireless communication systems faces the demand for increased data rates, higher mobility, larger carrier frequencies, and more link reliability. Wireless channels are characterized by fading, multipath, limited bandwidth, and frequency and time selectivity which make system design a challenge. It is therefore crucial to have an understanding of the behavior of wireless channels in order to know their performance limits and to be able to design efficient communication systems for them. This paper considers the analysis of the performance of digital communication systems with different coding and modulation schemes. Although digital communication is much better than the analog communication, still it has certain issues that need to be addressed. Especially when it comes to wireless communication, one of the major research considerations becomes the effect of multipath propagation. A thorough analysis is necessary for strategic planning of any system design by doing comparative study of different modulation techniques via different multipath communication channels. To study and draw the graph in terms Bit Error Rate (BER) versus $E_{b} /$ No in multipath communication channels for modulation schemes. Therefore, understand the system could go for more suitable modulation technique to suit the channel quality and can suggest better modulation schemes [2].

The performance of data transmission over wireless channels is well captured by observing their BER, which is a function of Signal to Noise Ratio (SNR) [5] at the receiver. In wireless channels, several models have been proposed and investigated to calculate SNR. All the models are a function of the distance between the sender and the receiver, the path loss exponent and the channel gain. Several probability distributed functions are available to model a time-variant parameter i.e. channel gain. We describe the three important and frequently used distributions. Those are Additive White Gaussian Noise (AWGN), Rayleigh and Rician models [9]. 
The remaining paper is organized as follows. In section II, channel models are described. In section III, fading is given. In section IV Modulation Techniques are explained and simulation results are given in section $\mathrm{V}$. The paper is concluded in section VI.

\section{Channel Model}

Wireless communication is now become an important part in our daily life and it is widely used in the technology development areas. Assembling of the various channels can be done accurately because the performance and the design of the channels depend upon the accuracy of the simulation. In the wireless communication field, fading is the important consideration because it tells about the fading patterns in the various conditions. There is no such model which tells about the environment. A signal that has chosen should be error free, or close to being error free [3]. If the signal is error free then the high quality of voice and data transmission can be done. The main issue arises while the development of the application is that the selection of the fading model. The analysis based on the DPSK will give the idea which helps for the application development in the market [4].

There are three main basic fading channel models i.e. Additive White Gaussian Noise (AWGN), Line of Sight (Rician) and Non Line of Sight (Rayleigh) Fading Channel models.

\subsection{Rayleigh Fading Channel}

Rayleigh fading occurs due to the multilink reception. In Rayleigh fading model the effect of the environment spreading to a larger area on a radio signal. It is one of the cheapest models of the signal propagation (i.e. for ionosphere and troposphere). Rayleigh fading is most applicable when there is no dominant propagation along a line of sight between transmitter and receiver. If the channel impulse response will be modeled as a Gaussian process with respect to the distribution of the individual components and if the process has zero mean and phase lie between 0 to $2 \pi$ radians [6]. Then, the probability density function can be given by:

$$
P_{R}(R)=\frac{R}{e^{2}} e-\frac{R^{2}}{2 \sigma^{2}}, 0 \leq R<\alpha
$$

\subsection{Rician Fading Channel}

Rician Fading is a part of Rayleigh fading with the introduction of a strong line of sight path in the Rayleigh fading environment. Rician fading is worthy for satellite communications and is acceptable for some urban scenarios. Rician fading is a type of small-scale fading because the probability of deep fades is less than that in the Rayleigh-fading case [6]. The probability density function of the amplitude is a Rician distribution and is mathematically expressed as follows:

$$
P_{R}(R)=\frac{R}{e^{2}} e-\frac{R^{2}+A^{2}}{\sigma^{2}} I_{0}\left(\frac{R A}{\sigma^{2}}\right), \quad 0 \leq R<\alpha
$$

\subsection{Additive White Gaussian Noise Channel}

For the case of Doppler Effect between a moving source and stationary receiver, narrowband data model is used to model the received signal at the antenna arrays. It presumes that the enclosure of the signal wave front inseminating across the antenna array necessarily remains constant [6]. This model is valid for the signals having bandwidth much smaller than the carrier frequency. According to above hypothesis, the received signal can be written as

$$
H(t)=A(\Theta) b(t)+N(t)
$$

Where, $\mathrm{A}(\Theta)$ is the array manifold vector and $\mathrm{N}(\mathrm{t})$ is AWGN with zero mean and two-sided power spectral density given by $\mathrm{N}_{0} / 2$.

\section{Fading}

Fading refers to the distortion that a carrier-modulated telecommunication signal experiences over certain propagation media. In wireless systems, fading is due to multipath propagation and is sometimes referred to as multipath induced fading. To understand fading, it is essential to understand multipath. In wireless telecommunications, multipath is the propagation phenomenon that results in radio signals' reaching the receiving antenna by two or more paths. Causes of multipath include atmospheric ducting, ionosphere reflection and refraction, and reflection from terrestrial objects, such as mountains and buildings. The effects of multipath include constructive and destructive interference, and phase shifting of the signal. This distortion of signals caused by multipath is known as fading. In other words it can be said that in the real world, multipath occurs when there is more than one path available for radio signal propagation. The phenomenon of reflection, diffraction and scattering all give rise to additional radio propagation paths beyond the direct optical LOS[7] (Line of Sight) path between the radio transmitter and receiver.

A Fading Channel is known as communications channel which has to face different fading phenomenon's, during signal transmission. In real world environment, the radio propagation effects combine together and multipath is generated by these fading channels. Due to multiple signal propagation paths, multiple signals will be received by receiver and the actual received signal level is the vector sum of the all signals. These signal incident from any direction or angle of arrival. In multipath, some signals aid the direct path and some others subtract it.

\subsection{Fading on the Basis of Effect of Multipath}

Large scale fading is defined as the fading which depends upon the location with respect to objects or it shows clearly in case of the short distance of the transmitter or the receiver. A continuous variation in the phase and amplitude occurs when a signal moves from a distance in the order of wavelength or it can also say that the small scale fading refer to the changes occur in the position of the transmitter and receiver in order of 
wavelength [8]. These changes are very small.

\subsection{Fading on the Basis of the Doppler Spread Effect}

Slow fading occurs when the minimum time required for the channel is large to change its magnitude from its previous value relative to the delay behavior of the channel. Slow fading can also be formed by shadowing. In shadowing, when large buildings or hills create problem for the path of the main signal of the transmitter and receiver, the received power is obtained by shadowing can be modeled by using log -distance path loss or log-normal distribution. The minimum time required for the channel is to change its magnitude from its previous value relative to the delay behavior of the channel is known as Fast fading.

\section{Modulation}

One way to communicate a message signal whose frequency spectrum does not fall within that fixed frequency range, or one that is unsuitable for the channel, is to change a transmittable signal according to the information in the message signal. This alteration is called modulation, and it is the modulated signal that is transmitted. The receiver then recovers the original signal through a process called demodulation.

Good bit error rate performance, less power consumption and good spectral efficiency are the Properties of modulation techniques. In digital system, the message signal is to be transmitted is digital in nature or we can say that the transmission of the information in digital form. There are various types of modulating schemes involves in the communication system like Phase shift keying(PSK), Frequency shift keying(FSK), Minimum shift keying(MSK), Quadrature phase shift keying(QPSK), Quadrature amplitude modulation(QAM) and Differential Phase Shift Keying (DPSK).

\subsection{Differential Phase Shift Keying}

Differential phase shift keying (DPSK) [9], a common form of phase modulation conveys data by changing the phase of carrier wave. In Phase shift keying, High state contains only one cycle but DPSK contains one and half cycle. Differential Shift Keying is a modulation technique that codes information by using the phase difference between two neighboring symbols. In the transmitter, each symbol is modulated relative to the previous symbol and modulating signal, for instance in BPSK 0 represents no change and 1 represents +180 degrees. In the receiver, the current symbol is demodulated using the previous symbol as a reference. The previous symbol serves as an estimate of the channel. A no change condition causes the modulated signal to remain to remain at the same 0 or 1 state of the previous symbol. Differential modulation is theoretically $3 \mathrm{~dB}$ poorer than coherent. This is because the differential system has 2 sources of error: a corrupted symbol, and a corrupted reference.

In DPSK, the transmitter, each symbol is modulated relative to the phase of the immediately preceding signal element and the data being transmitted. In this paper, we choose M-DPSK where $\mathrm{M}=2,4,8,16,32,64$ scheme to analyze SNR with constant BER in different fading channels.

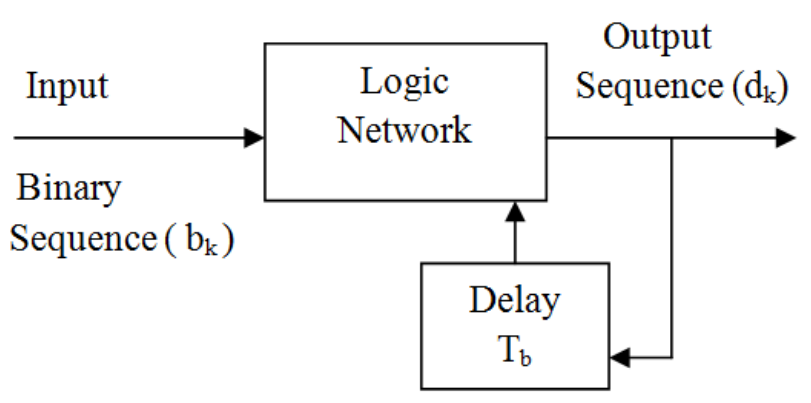

Figure 1. Block Diagram of DPSK Modulation.

\subsection{Bit Error Rate (BER)}

The BER or quality of the digital link is calculated from the number of bits received in error divided by the number of bits transmitted.

\section{$\mathrm{BER}=($ Bits in Error $) /$ (Total bits received).}

In digital transmission, the number of bit errors is the number of received bits of a data stream over a communication channel that has been altered due to noise, interference, distortion or bit synchronization errors. The BER is the number of bit errors divided by the total number of transferred bits during a particular time interval. BER is a unit less performance measure, often expressed as a percentage.

For example, $\mathrm{N}$ erroneous bits out of 1000 bits transmitted would be expressed as $\mathrm{N}^{*} 10^{-3}$. In this paper we assume that one erroneous bit out of 1000 bits would be transmitted. That is the bit error rate is $1 * 10^{-3}$.

\subsection{Signal to Noise Ratio (SNR)}

SNR is the ratio of the received signal strength over the noise strength in the frequency range of the operation. It is an important parameter of the physical layer of Local Area Wireless Network (LAWN). Noise strength, in general, can include the noise in the environment and other unwanted signals (interference). BER is inversely related to SNR, that is high BER causes low SNR. High BER causes increases packet loss, increase in delay and decreases throughput. The exact relation between the SNR and the BER is not easy to determine in the multi channel environment. Signal to noise ratio (SNR) is an indicator commonly used to evaluate the quality of a communication link and measured in decibels and represented by Eq. (4).

$$
\mathrm{SNR}=10 \log _{10}(\text { Signal Power } / \text { Noise Power }) \mathrm{dB}
$$

SNR measures the quality of a transmission over a network channel. The grater the signal to noise ratio, the easier it is to identify and subsequently isolate and eliminate the source of noise. A SNR of zero indicates that the desired signal is virtually indistinguishable from the unwanted noise. 
In this paper, we tested various fading channel in accordance with SNR with constant bit error rate, in our case which is $10^{-3}$.

\section{Simulation and Results}

In this paper, one of the important topics in wireless communication, which is the concept of fading, is demonstrated by the approach available in MATLAB [10].

A wireless Communication system was designed in MATLAB. It was assumed the data was first encoded with linear block coding and then was transmitted thought the channel. The transmitted signal is distorted by noise which was assumed as Additive white Gaussian noise. The fading effect was also taken in consideration. So, different fading channel mode was considered.

The important topic in wireless communications, that is the concept of fading, is demonstrated by the approach available in MATLAB. One of the important aspects of the path between the transmitter and receiver is the occurrence of fading. MATLAB provides a simple and easy way to demonstrate fading taking place in wireless systems. Statistical testing can subsequently be used to establish the validity of the fading models frequently used in wireless systems. The different fading models and MATLAB based simulation approaches will now be described.

In order to be statistically significant, each simulation must generate some number of errors. If any channel does not contain any noise the channel capacity will be infinite (according to Shannon's channels capacity theorem) in practice no channel can be noise free. In this simulation the test signal contains 1000 bits and receiver will identify only one bit error out of 1000 bits, that is bit error rate is $0.1 \%$ since a bit error rate of one percent is considered quiet high, that's why we consider BER much lower than $1 \%$.

The simulation is followed by using $\mathrm{m}$ file. In this approach, the simulation is successfully done using DPSK modulation technique. The desired BER versus SNR are obtained for simulation in AWGN, Rayleigh and Rician channels.

\subsection{Transmission through AWGN Channel}

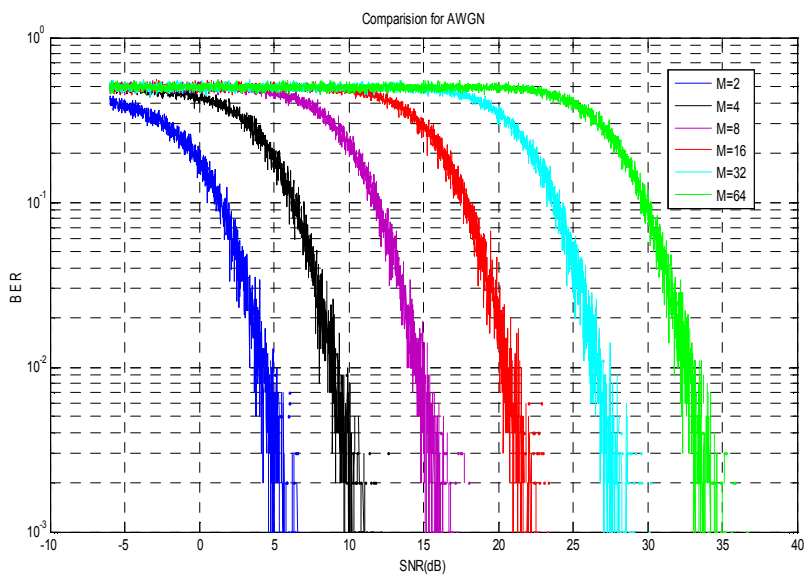

Figure 2. Bit Error Rate vs. SNR over AWGN channel for 2, 4, 8, 16, 32, 64 DPSK.
Figure 2 shows Bit Error Rate vs. SNR over AWGN channel for 2, 4, 8, 16, 32, 64 DPSK.

\subsection{Transmission through Rayleigh Channel}

Figure 3 shows Bit Error Rate vs. SNR over Rayleigh channel for 2, 4, 8, 16, 32, 64 DPSK.

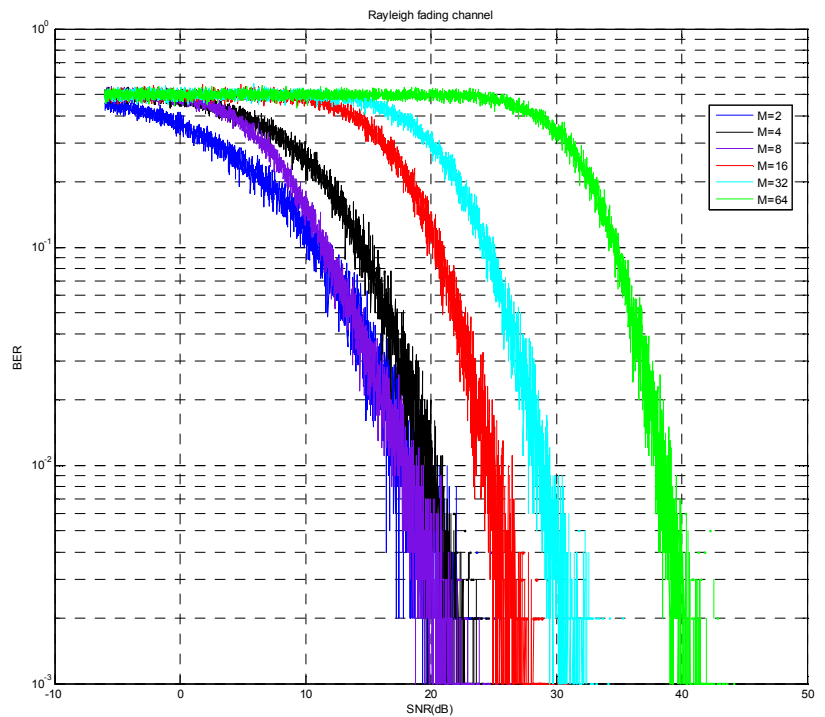

Figure 3. Bit Error Rate vs. SNR over Rayleigh channel for 2, 4, 8, 16, 32, 64 DPSK modulations.

\subsection{Transmission through Rician Channel}

Figure 4 shows Bit Error Rate vs. SNR over Rician channel for 2, 4, 8, 16, 32, 64 DPSK.

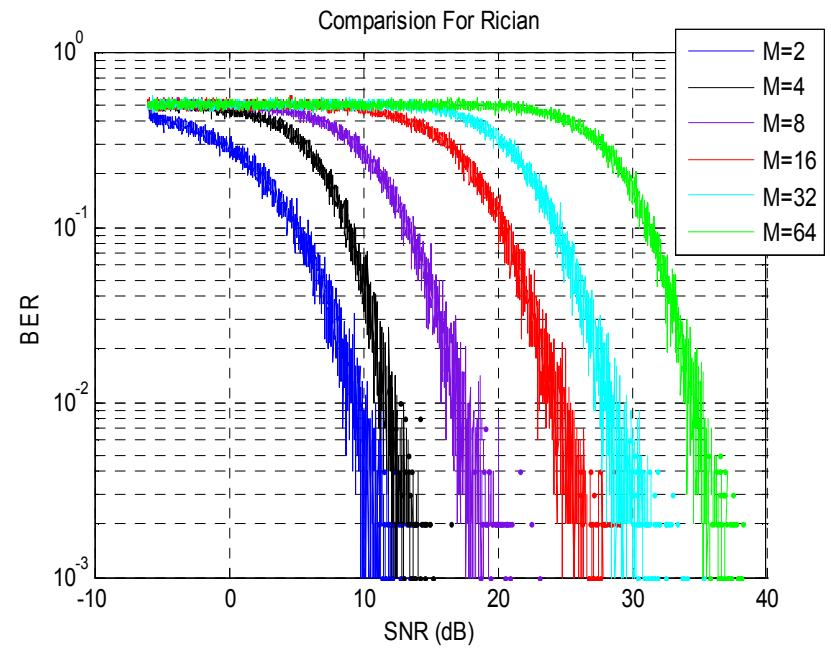

Figure 4. Bit Error Rate vs. SNR over Rician channel for 2, 4, 8, 16, 32, 64 DPSK modulations.

We can understand from the above figure 2, 3 and 4 that, the value of SNR is rising with the higher value of $M$ for the constant value of BER which is $10^{-3}$. If the value of $\mathrm{M}$ is increased, the SNR will be increased and for that reason the transmitted signal will be distorted and it will be time consuming and costly to recover the original signal. 


\subsection{Comparison Transmission between AWGN, Rayleigh and Rician Channel}

Figure 5 shows Bit Error Rate vs. SNR over AWGN, Rayleigh and Rician channel for 4 DPSK modulations.

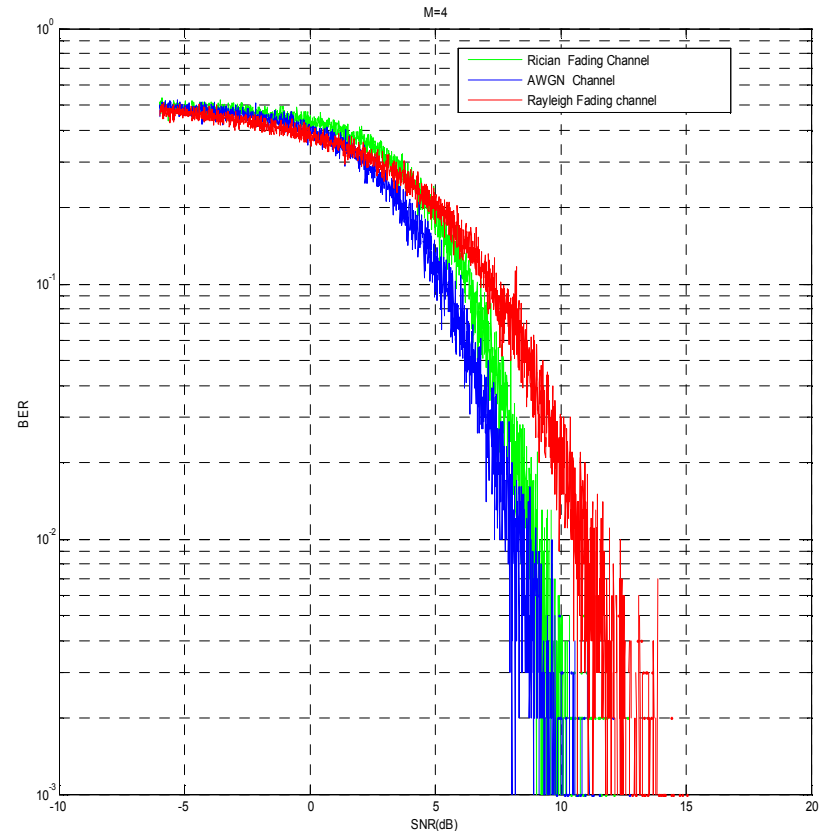

Figure 5. Bit Error Rate vs. SNR over AWGN, Rayleigh and Rician channel for 4 DPSK modulations.

Figure 6 shows Bit Error Rate vs. SNR over AWGN, Rayleigh and Rician channel for 16 DPSK modulations.

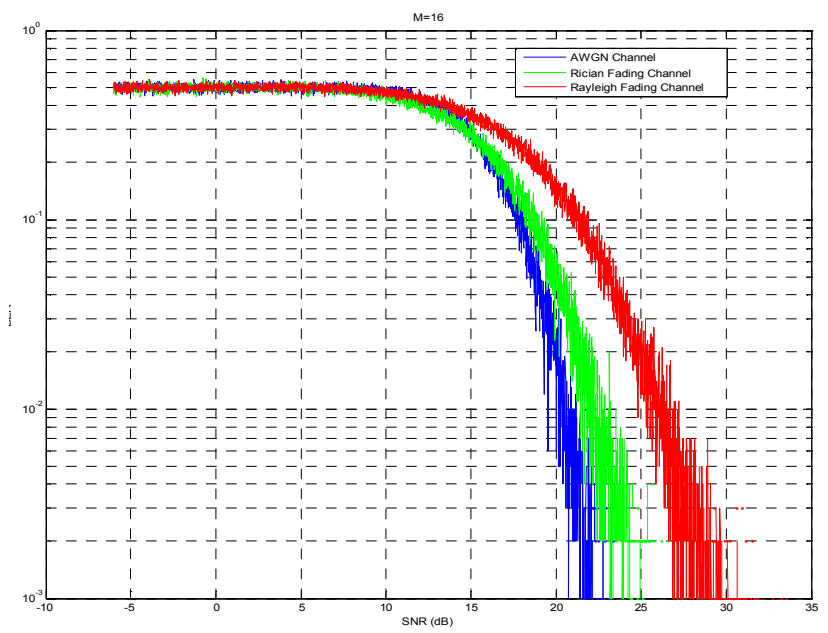

Figure 6. Bit Error Rate vs. SNR over AWGN, Rayleigh and Rician channel for 16 DPSK modulations.

Figure 7 shows Bit Error Rate vs. SNR over AWGN, Rayleigh and Rician channel for 64 DPSK modulations.

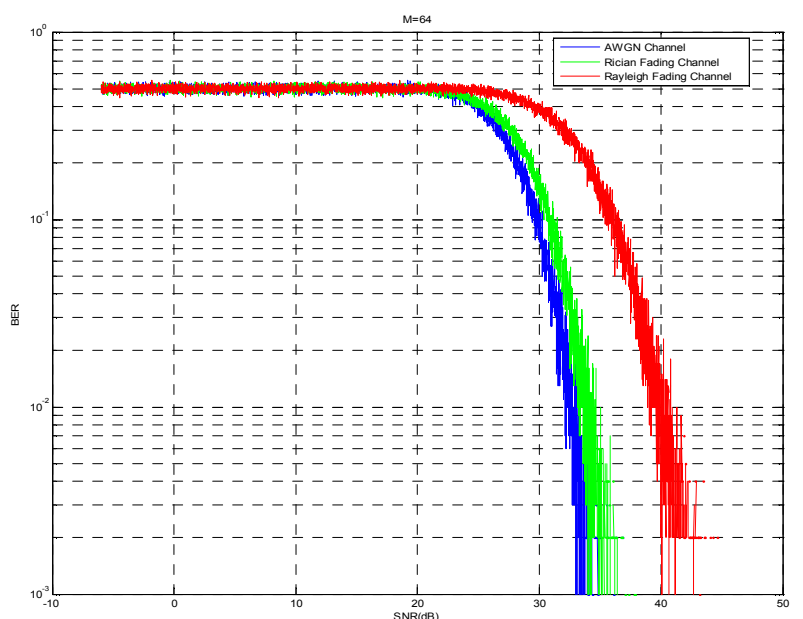

Figure 7. Bit Error Rate vs. SNR over AWGN, Rayleigh and Rician channel for 64 DPSK modulations.

It has seen from the figure 5, 6 and 7 that, for $M=4,16$ and 64 the demodulated signals of different AWGN, Rayleigh and Rician Fading Channel are almost like waterfall with a different SNR value. AWGN channel is not a fading channel, so the BER vs. SNR rate will be compared with Rayleigh and Rician Fading channel. From those channels Rician Channel has both LOS (Line of Sight) and multipath, so the SNR value for Rician will definitely lower than the Rayleigh fading channel for the direct transmission path. The Rician Channel is much better for signal transmission compared with the Rayleigh fading channel.

Table 1. BER vs. SNR Comparison for AWGN, Rayleigh and Rician Channel.

\begin{tabular}{lllll}
\hline \multirow{2}{*}{ BER } & \multirow{2}{*}{ Values of $\mathbf{M}$} & \multicolumn{2}{l}{ SNR(dB) } & \\
\cline { 3 - 5 } & & AWGN & Rayleigh & Rician \\
\hline $10^{-3}$ & 4 & 10 & 14 & 12 \\
$10^{-3}$ & 16 & 22 & 28 & 24 \\
$10^{-3}$ & 64 & 32 & 42 & 36 \\
\hline
\end{tabular}

For DPSK, various values of SNR with constant BER $10^{-3}$ are obtained using different values of $\mathrm{M}$ shown in the table 1. When the value of $\mathrm{M}$ is $4, \mathrm{SNR}$ is $10 \mathrm{~dB}$ for AWGN, $14 \mathrm{~dB}$ for Rayleigh, $12 \mathrm{~dB}$ for Rician. When the value of $\mathrm{M}$ is $16, \mathrm{SNR}$ is $22 \mathrm{~dB}$ for AWGN, $28 \mathrm{~dB}$ for Rayleigh, $24 \mathrm{~dB}$ for Rician. For the value of $\mathrm{M}$ equal to 64 , SNR is $32 \mathrm{~dB}$ for $\mathrm{AWGN}, 42 \mathrm{~dB}$ for Rayleigh, $36 \mathrm{~dB}$ for Rician.

Rayleigh fading channel has comparatively worst performance with compared to AWGN and Rician fading channel. This is because Rayleigh fading has no LOS from transmitter to receiver.

\section{Conclusion}

In Wireless communication system, the needs of data rate are increasing day by day which requires more bit rate in same channel bandwidth. Multi level digital modulation schemes are the one of such techniques by which it is possible to transmit more bit rate in same channel. In such modulation technique, for $k$-bits of information, one of the $M=2^{k}$ possible symbols is used to modulate a carrier signal, which results of 
$k$-times increment of bit rate in same bandwidth.

From simulation results, it is seen that for same values of $\mathrm{M}$ the Rician channel exhibits better performance than AWGN and Rayleigh in terms of SNR with constant BER.

\section{References}

[1] Swamy M. Katta, Deepthi M., et.al, "Performance Analysis of DSSS and FHSS Techniques over AWGN Channel", International Journal of Advancements in Technology, Volume 4, No. 1, March 2013.

[2] Fating Pooja P., Ashtankar Pankaj S., "Comparative Study of Different Modulation Techniques for Multipath Communication Channel", Proceedings of 4th SARC International Conference, Nagpur, India, ISBN: 978-93-82702-70-2 March 30th, 2014.

[3] Mohammaed Slim Alouini and Andrea J. Goldsmith "Capacity of Rayleigh fading channels under different Adaptive Transmission and Diversity combining Techniques", IEEE Transactions on Vehicular Technology, Vol. 48, No. 4, July 1999.

[4] Gary Breed, High Frequency Electronics, 2003 Summit, Technical Media LLC "Bit Error Rate: Fundamental Concepts and measurement issues".

[5] Fumiyaki Adachi, "error Rate Analysis of Differentially Encoded and detected 16-APSK under Rician fading", IEEE Transactions on Vehicular Technology, Vol. 45, No. 1, February 1996.

[6] Mohammaed Slim Alouini and Andrea J. Goldsmith “ Capacity of Rayleigh fading channels under different Adaptive Transmission and Diversity combining Techniques", IEEE Transactions on Vehicular Technology, Vol. 48, No. 4, July 1999.
[7] Gupta, Akhil. "Improving Channel Estimation in OFDM System Using Time Domain Channel Estimation for Time Correlated Rayleigh Fading Channel Model." International Journal of Engineering and Science Invention, vol. 2, issue 8, pp. 45-51, August. 2013

[8] "Quadrature Amplitude Modulation", digital Modulation Techniques" www.digitalmodulation.net/qam.html

[9] Kaur Harjot \& Verma Amit, "BER Performance Analysis of Mary DPSK Techniques Using Simulation Modelling", International Journal of Electrical and Electronics Engineering Research (IJEEER), ISSN 2250-155X Volume 3, Issue 2, Jun 2013, pp. 93-100.

[10] James E. Gilpy, Transcript International Inc., August 2003, "Bit Error Rate Simulation using Matlab".

[11] J. P. E. Biglieri and S. Shamai, "Fading Channels: Information Theoretic and Communications Aspects," IEEE Trans. Inform. Theory, vol. 44, no. 6, pp. 2619-2692, Oct. 1998

[12] Pandey Ashish, Singh Sarala et.al, "Comparative Study of Different Modulation Technique in Chaotic Communication", International Journal of Scientific Research Engineering \& Technology (IJSRET), Volume 2, Issue 11, February 2014, pp 738-743.

[13] Dixit Dutt Bohra, Avnish Bora," Bit Error Rate Analysis in Simulation of Digital Communication Systems with Different Modulation Schemes", IJISET - International Journal of Innovative Science, Engineering \& Technology, Vol. 1 Issue 3, May 2014.

[14] A. Sudhir Babu and Dr. K.V Sambasiva Rao," Evaluation of BER for AWGN, Rayleigh and Rician Fading Channels under Various Modulation Schemes", International Journal of Computer Applications (0975 - 8887) Volume 26- No.9, July 2011. 